\title{
Anomalies and Tadpoles in Open/Closed String Duality
}

\author{
A. Liccardo, R. Marotta and F. Pezzella \\ Dipartimento di Scienze Fisiche, Università di Napoli and INFN, Sezione di Napoli \\ Complesso Universitario Monte S. Angelo, ed. G - via Cintia - I-80126 Napoli, Italy
}

\begin{abstract}
We discuss the role played by the divergences appearing in the interaction between a fractional D3 brane dressed with an $S U(N)$ gauge field and a stack of $N$ fractional D3 branes on the orbifolds $C^{2} / Z_{2}$ and $C^{3} /\left(Z_{2} \times Z_{2}\right)$. In particular we show that the logarithmic divergences in the closed string channel, interpreted as due to twisted massless tadpoles, are mapped, under open/closed string duality, in the logarithmic ones in the open string channel, due to the massless states circulating in the annulus diagram and corresponding to the one-loop divergences that one finds in the gauge theory living in the world volume of the brane. This result provides a quantitative evidence of why the chiral and scale anomalies of the supersymmetric and non conformal gauge theories supported by the world volume of the branes can be inferred from supergravity calculations.
\end{abstract}

Work partially supported by the European Commission RTN Programme HPRN-CT-2000-00131 and by MIUR. 


\section{Introduction}

This work, based on Ref. [1], is aimed to a deeper understanding of the relation between the gauge/gravity correspondence and the open/closed string duality. The former indicates the two-fold property exhibited by a D-brane of being a solution of the low-energy string effective action (supergravity) and of having open strings with their endpoints attached to its world-volume; the latter denotes the equivalence between the open string annulus diagram, parametrized by the proper time $\tau$, and the closed string tree diagram, when the modular transformation $\tau \rightarrow 1 / \tau$ is performed - for recent reviews on this subject, see for example Ref. [2].

By using the gauge/gravity correspondence it has been shown that the classical supergravity solutions corresponding to fractional D branes, stuck at the orbifold fixed points, encode perturbative properties of non conformal gauge theories, with reduced supersymmetry, living on their world volume, such as the chiral and scale anomaly ${ }^{1}$. Relevant improvements have been performed in deriving from such a kind of branes also nonperturbative properties of the gauge theories [5] [6]. Since, as it is well-known, gauge theories and supergravity are related to open and closed strings respectively, it is quite natural to investigate on the possibility of deriving the gauge theory anomalies by only using the open/closed string duality in a pure stringy framework. In other words, one can ask whether the gauge/gravity correspondence could be seen as a direct consequence of the open/closed string duality. The answer we have got is positive, at least in the cases we have considered. More precisely, we can state that the quantum ultraviolet properties of the gauge theory living on a D-brane can be equivalently derived by performing the field theory limit either in the open string channel, as expected, or in the closed string channel and in this case the gauge theories anomalies can be inferred from logarithmic divergences due to the exchange of twisted massless states (twisted tadpoles). Hence a clear and beautiful relation between the gauge/gravity correspondence and the open/closed string duality, lying in turn on the relation between the gauge theory anomalies and the presence of twisted massless states in the closed string channel, emerges out. This has been the main goal of our work.

Our starting point has been the computation of the one-loop string annulus diagram which describes the interaction of a fractional brane of the orbifold $C^{2} / Z_{2}$ having an $S U(N)$ gauge field on it with $N$ fractional branes without any gauge field and we have extracted from it the coefficient of the gauge kinetic term. This contribution results to be logarithmically divergent at the string level [7] [8] 9]. But, differently from other kinds of higher divergences which are in general a sign of inconsistency of the theory and therefore have to be eliminated in some way (see for instance Refs. [10] and [11]), this logarithmic divergence can be interpreted, when read from the point of view of the closed string channel, as due to the exchange of twisted massless tadpoles. The logarithmic nature of

\footnotetext{
${ }^{1}$ For a review of fractional branes and their application to the study of non conformal gauge theories see Refs. [3] and 4].
} 
this divergence derives from the fact that such an exchange occurs in the two directions transverse to the branes and the orbifold. Furthermore this divergence is ultraviolet in the open string channel and infrared in the closed string one, therefore we have regularized the string calculation by introducing an ultraviolet cutoff in the former corresponding to an infrared cutoff in the latter. We have shown that, under the modular transformation that maps the open string channel into the closed string one, open string massless states go into closed string massless states, and open string massive states go into closed string massive states, without any mixing between massless and massive states! Hence the divergent contribution can be seen to come from the exchange of massless closed string states between the two fractional branes or from the massless open string states circulating in the loop. By adding also the contribution of the open string tree diagrams to the one-loop string diagram, we find an expression for the gauge coupling constant that gives the correct beta-functions of $\mathcal{N}=2$ and $\mathcal{N}=1$. This result provides a quantitative evidence of why the one loop-beta function can be derived from supergravity calculations [12, [13, 14] 15] [16] 17] 18 .

\section{Branes in External Fields: $\mathcal{N}=2$ orbifold}

We analyze the interaction between a D3 brane with an external $S U(N)$ gauge field on its world-volume and a stack of $N$ ordinary D3 branes. This can be equivalently done by computing either, in the open string channel, the one-loop open string diagram or, in the closed string channel, the tree closed string diagram containing two boundary states and a closed string propagator.

In order to obtain a gauge theory with reduced supersymmetry we consider Type IIB superstring theory on an orbifold space. Furthermore, to get a non conformal gauge theory, we study fractional D3 branes which are characterized by their being stuck at the orbifold fixed point and which, unlike bulk branes, have a non conformal theory on their world-volume. For the sake of simplicity we consider fractional branes of the orbifold $\mathbb{R}^{1,5} \times C^{2} / Z_{2}$ supporting $\mathcal{N}=2$ super Yang-Mills on their world volume.

The $Z_{2}$ group, that is chosen to be acting on the coordinates $x^{m}$ with $m=6,7,8,9$, is characterized by two elements $(e, h)$, being $e$ the identity element and $h$ such that $h^{2}=e$. The element $h$ acts on the complex combinations $\vec{z}=\left(z^{1}, z^{2}\right)$, where $z^{1}=x^{6}+i x^{7}$, $z^{2}=x^{8}+i x^{9}$, as $\left(z_{1}, z_{2}\right) \rightarrow\left(-z_{1},-z_{2}\right)$. The orbifold group $Z_{2}$ acts also on the ChanPaton factors located at the endpoints of the open string stretched between the branes. Fractional branes are defined as branes for which such factors transform according to irreducible representations of the orbifold group and we consider only the trivial one corresponding to a particular kind of fractional branes. The orbifold we are considering is non compact and has therefore only one fixed point located at $z_{1}=z_{2}=0$. We are interested in the case of parallel fractional D3 branes with their world volume along the directions $x^{0}, x^{1}, x^{2}, x^{3}$, that are completely external to the space on which the orbifold acts. The gauge field lives on the four-dimensional world volume of the fractional D3 brane 
and can be chosen to have the following non vanishing entries:

$$
\hat{F}_{01}=-\hat{F}_{10}=f \quad \hat{F}_{23}=-\hat{F}_{32}=g,
$$

where $\hat{F}_{\alpha \beta}=2 \pi \alpha^{\prime} F_{\alpha \beta}$.

The interaction between two branes is given by the vacuum fluctuation of an open string that is stretched between them. In particular, the free energy of an open string stretching between a D3 brane and a stack of $N$ D3 branes located at a distance $y$ in the plane $\left(x^{4}, x^{5}\right)$, orthogonal to both the D3 brane world-volume and the four-dimensional space on which the orbifold acts, is given by [1]:

$$
\begin{aligned}
Z_{h}^{o} & =\frac{N}{\left(8 \pi^{2} \alpha^{\prime}\right)^{2}} \int d^{4} x \sqrt{-\operatorname{det}(\eta+\hat{F})} \int_{0}^{\infty} \frac{d \tau}{\tau} e^{-\frac{y^{2} \tau}{2 \pi \alpha^{\prime}}} \frac{4 \sin \pi \nu_{f} \sin \pi \nu_{g}}{\Theta_{2}^{2}(0 \mid i \tau) \Theta_{1}\left(i \nu_{f} \tau \mid i \tau\right) \Theta_{1}\left(i \nu_{g} \tau \mid i \tau\right)} \\
& \times\left[\Theta_{3}^{2}(0 \mid i \tau) \Theta_{4}\left(i \nu_{f} \tau \mid i \tau\right) \Theta_{4}\left(i \nu_{g} \tau \mid i \tau\right)-\Theta_{4}^{2}(0 \mid i \tau) \Theta_{3}\left(i \nu_{f} \tau \mid i \tau\right) \Theta_{3}\left(i \nu_{g} \tau \mid i \tau\right)\right] \\
& +\frac{i N}{32 \pi^{2}} \int d^{4} x F_{\alpha \beta}^{a} \tilde{F}^{a \alpha \beta} \int_{0}^{\infty} \frac{d \tau}{\tau} e^{-\frac{y^{2} \tau}{2 \pi \alpha^{\prime}}},
\end{aligned}
$$

where $\tilde{F}_{\alpha \beta}=\frac{1}{2} \epsilon_{\alpha \beta \gamma \delta} F^{\gamma \delta}$ and

$$
\nu_{f} \equiv \frac{1}{2 \pi i} \log \frac{1+f}{1-f} \quad \text { and } \quad \nu_{g} \equiv \frac{1}{2 \pi i} \log \frac{1-i g}{1+i g} .
$$

Here we have only considered the term in the free energy expression coming from the non trivial element $h$ of the orbifold group. Indeed this is the only one that contributes to the one-loop anomalies of the gauge theory in the world volume, as we will see later.

The above computation can also be performed in the closed string channel where the relevant contribution is due to the tree level propagation of twisted states, that is:

$$
\begin{aligned}
Z_{h}^{c} & =\frac{N}{\left(8 \pi^{2} \alpha^{\prime}\right)^{2}} \int d^{4} x \sqrt{-\operatorname{det}(\eta+\hat{F})} \int_{0}^{\infty} \frac{d t}{t} e^{-\frac{y^{2}}{2 \pi \alpha^{\prime} t}} \frac{4 \sin \pi \nu_{f} \sin \pi \nu_{g}}{\Theta_{4}^{2}(0 \mid i t) \Theta_{1}\left(\nu_{f} \mid i t\right) \Theta_{1}\left(\nu_{g} \mid i t\right)} \\
& \times\left\{\Theta_{2}^{2}(0 \mid i t) \Theta_{3}\left(\nu_{f} \mid i t\right) \Theta_{3}\left(\nu_{g} \mid i t\right)-\Theta_{3}^{2}(0 \mid i t) \Theta_{2}\left(\nu_{f} \mid i t\right) \Theta_{2}\left(\nu_{g} \mid i t\right)\right\} \\
& +\frac{i N}{32 \pi^{2}} \int d^{4} x F_{\alpha \beta}^{a} \tilde{F}^{a \alpha \beta} \int \frac{d t}{t} e^{-\frac{y^{2}}{2 \pi \alpha^{\prime} t}} .
\end{aligned}
$$

The two expressions for $Z$ separately obtained in the open and the closed string channels are, as expected, equal to each other. This equality is the essence of the open/closed string duality and can be explicitly shown by using the modular transformation that relates the modular parameters in the open and closed string channels, namely $\tau=1 / t$. It is also easy to see that the distance $y$ between the dressed D3 brane and the stack of the $N$ D3 branes makes the integral in Eq. (4) convergent for small values of $t$, while in the limit $t \rightarrow \infty$, the integral is logarithmically divergent. This divergence is due to a twisted tadpole corresponding to the exchange of massless closed string states between the two stucks of branes. We would like to stress that the presence of the gauge field makes the divergence to appear already at the string level, before any field theory limit $\left(\alpha^{\prime} \rightarrow 0\right)$ is performed. When $F$ vanishes, the divergence is eliminated by the integrand being identically zero as a consequence of the fact that fractional branes are BPS states. 
Tadpole divergences correspond in general to the presence of gauge anomalies, which make the gauge theory inconsistent and must be eliminated by drastically modifying the theory or by fixing particular values of the parameters. Instead, as stressed in Refs. 7] 8] 19] 20], logarithmic tadpole divergences do not correspond to gauge anomalies. In our case they correspond to the fact that the gauge theory living on the brane is not conformally invariant. In fact, they provide the correct one-loop running coupling constant. We cure these divergences just by introducing in Eq. (4) an infrared cutoff $\Lambda$ that regularizes the contribution of the massless closed string states. Since, in the open/closed string duality, an infrared divergence in the closed string channel corresponds to an ultraviolet divergence in the open string channel, it is easy to see that the expression in Eq. (2) is divergent for small values of $\tau$ and needs an ultraviolet cutoff. This divergence is exactly the one-loop divergence that one gets in $\mathcal{N}=2$ super Yang-Mills, which is the gauge theory living in the world volume of the fractional D3 brane.

From Eqs. (2) and (4), which contain arbitrary powers of the gauge field $F$, we extract the quadratic term in $F$. This term in the open string channel is given by:

$$
\begin{aligned}
Z_{h}^{o}(F) & \rightarrow\left[-\frac{1}{4} \int d^{4} x F_{\alpha \beta}^{a} F^{a \alpha \beta}\right] \\
& \times\left\{\frac{1}{g_{Y M}^{2}(\Lambda)}-\frac{N}{8 \pi^{2}} \int_{\frac{1}{\alpha^{\prime} \Lambda^{2}}}^{\infty} \frac{d \tau}{\tau} e^{-\frac{y^{2} \tau}{2 \pi \alpha^{\prime}}}+\frac{N}{8 \pi^{2}} \int_{0}^{\infty} \frac{d \tau}{\tau} e^{\left.-\frac{y^{2} \tau}{2 \pi \alpha^{\prime}} G(k)\right\}}\right. \\
& +i N\left[\frac{1}{32 \pi^{2}} \int d^{4} x F_{\alpha \beta}^{a} \tilde{F}^{a \alpha \beta}\right] \int_{\frac{1}{\alpha^{\prime} \Lambda^{2}}}^{\infty} \frac{d \tau}{\tau} e^{-\frac{y^{2} \tau}{2 \pi \alpha^{\prime}}}
\end{aligned}
$$

where we have isolated the massless open string states, which are the only ones giving a divergence, from the massive states encoded in the function $G(k)$ so defined:

$$
G(k)=-\left[\frac{f_{3}(k) f_{4}(k)}{f_{1}(k) f_{2}(k)}\right]^{4} 2 k \frac{d}{d k} \log \left[\frac{f_{3}(k)}{f_{4}(k)}\right]+1,
$$

Analogously in the closed channel one gets:

$$
\begin{aligned}
Z_{h}^{c}(F) & \rightarrow\left[-\frac{1}{4} \int d^{4} x F_{\alpha \beta}^{a} F^{a \alpha \beta}\right] \\
& \times\left\{\frac{1}{g_{Y M}^{2}(\Lambda)}-\frac{N}{8 \pi^{2}} \int_{0}^{\alpha^{\prime} \Lambda^{2}} \frac{d t}{t} e^{-\frac{y^{2}}{2 \pi \alpha^{\prime} t}}+\frac{N}{8 \pi^{2}} \int_{0}^{\infty} \frac{d t}{t} e^{-\frac{y^{2}}{2 \pi \alpha^{\prime} t}} F(q)\right\} \\
& +i N\left[\frac{1}{32 \pi^{2}} \int d^{4} x F_{\alpha \beta}^{a} \tilde{F}^{a \alpha \beta}\right] \int_{0}^{\alpha^{\prime} \Lambda^{2} c} \frac{d t}{t} e^{-\frac{y^{2}}{2 \pi \alpha^{\prime} t}}
\end{aligned}
$$

where, again, the massive states contribute as:

$$
F(q)=\left[\frac{f_{3}(q) f_{2}(q)}{f_{1}(q) f_{4}(q)}\right]^{4} 2 q \frac{d}{d q} \log \left[\frac{f_{3}(q)}{f_{2}(q)}\right]+1 .
$$

It turns out that the divergence that we have at the string level is exactly the one-loop divergence present in the gauge field theory living in the world volume of the brane and can be equivalently seen as due, in the open channel, to the massless open string states 
circulating in the loop and, in the closed channel, to the massless closed string states exchanged between two branes.

Notice that in the two previous equations we have also added the contribution coming from the tree diagrams that contain the bare coupling constant. In an ultraviolet finite theory such as string theory we should not deal with a bare and a renormalized coupling. On the other hand, we have already discussed the fact that the introduction of a gauge field produces a string amplitude that is divergent already at the string level and that therefore must be regularized with the introduction of a cutoff.

One can see that the contribution of the massless states and the one of the massive states transform respectively into each other without any mixing between massless and massive states, as follows from the identity:

$$
F(q)=G(k)
$$

that can be easily proven using the modular transformations of the functions $f_{i}$.

This means that the open/closed string duality exactly maps the ultraviolet divergent contribution coming from the massless open string states circulating in the loop - and that reproduces the divergences of $\mathcal{N}=2$ super Yang-Mills living in the world volume of the fractional D3 branes - into the infrared divergent contribution due to the massless closed string states propagating between the two branes. This leads to the first evidence of why the one-loop running coupling constant can be consistently derived from a supergravity calculation.

To show more explicitly the connections between open/closed duality and gauge/gravity correspondence we perform the field theory limit of the previous expressions. This means to perform the zero slope limit $\left(\alpha^{\prime} \rightarrow 0\right)$ together with the limit in which the modular variables $t$ and $\tau$ go to infinity, keeping fixed the dimensional Schwinger proper times $\sigma=\alpha^{\prime} \tau$ and $s=\alpha^{\prime} t$ of the open and closed string, respectively. Indeed, in these two limits the massive states contributions in Eqs. (5) and (77) - depending on $G(k)$ and $F(q)$ respectively - vanish leaving only the divergences due to the massless states. By extracting the coefficient of the term $F^{2}$ from either of the two Eqs. (5) and (77) one obtains:

$$
\frac{1}{g_{Y M}^{2}(\epsilon)}+\frac{N}{8 \pi^{2}} \log \frac{y^{2}}{\epsilon^{2}} \equiv \frac{1}{g_{Y M}^{2}(y)} \quad, \quad \epsilon^{2} \equiv 2 \pi\left(\alpha^{\prime} \Lambda\right)^{2}
$$

Eq. (10) gives the one-loop correction to the bare gauge coupling constant $g_{Y M}(\Lambda)$ in the gauge theory regularized with the cutoff $\Lambda$. By performing the renormalization procedure one gets the renormalized coupling constant:

$$
\frac{1}{g_{Y M}^{2}(\mu)}-\frac{N}{8 \pi^{2}} \log \frac{\mu^{2}}{m^{2}}=\frac{1}{g_{Y M}^{2}(m)} \quad ; \quad m^{2} \equiv \frac{y^{2}}{2 \pi \alpha^{\prime 2}} .
$$

from which one can determine the one-loop $\beta$-function of $\mathcal{N}=2$ super Yang-Mills.

The vacuum angle $\theta_{Y M}$ is provided by the terms in Eqs. (2) and (44) with the topological charge. If we extract it from either of these two equations, we find that it is imaginary 
and, moreover, must be renormalized like the coupling constant. A way of eliminating these problems is to introduce a complex cutoff and to consider both the configurations in which the gauge field is in either one of the two sets of branes. By taking the symmetric combination of these two configurations and introducing a complex cutoff $\Lambda \rightarrow \Lambda e^{-i \theta}$, the divergent integral in Eq. (5), or equivalently in Eq. (7), becomes:

$$
I(z) \equiv \int_{1 / \Lambda^{2}}^{\infty} \frac{d \sigma}{\sigma} e^{-\frac{y^{2} \sigma}{2 \pi\left(\alpha^{\prime}\right)^{2}}} \simeq \log \frac{2 \pi\left(\alpha^{\prime}\right)^{2} \Lambda^{2}}{y^{2} e^{2 i \theta}} .
$$

This procedure leaves unchanged all the previous considerations concerning the gauge coupling constant, because in this case the coefficient of the $F^{2}$ term results to be proportional to the following combination:

$$
\frac{1}{2}[I(z)+I(\bar{z})] \simeq \log \frac{2 \pi\left(\alpha^{\prime}\right)^{2} \Lambda^{2}}{y^{2}}
$$

In the case of the $\theta_{Y M}$ angle one gets instead:

$$
\theta_{Y M}=i \frac{N}{2}[I(z)-I(\bar{z})]=2 N \theta
$$

exactly reproducing the $\mathcal{N}=2 \mathrm{SYM}$ chiral anomaly.

\section{Branes in External Fields: $\mathcal{N}=1$ orbifold}

The analysis performed in the previous Section has been extended to the case of the orbifold $C^{3} /\left(Z_{2} \times Z_{2}\right)$ preserving four supersymmetry charges [1]. The orbifold group $Z_{2} \times Z_{2}$ contains four elements whose action on the three complex coordinates

$$
z_{1}=x_{4}+i x_{5} \quad z_{2}=x_{6}+i x_{7} \quad z_{3}=x_{8}+i x_{9}
$$

is chosen to be:

$$
\begin{aligned}
& R_{e}=\operatorname{diag}(1,1,1) \quad R_{h_{1}}=\operatorname{diag}(1,-1,-1) \\
& R_{h_{2}}=\operatorname{diag}(-1,1,-1) \quad R_{h_{3}}=\operatorname{diag}(-1,-1,+1) .
\end{aligned}
$$

The group $Z_{2} \times Z_{2}$ has four irreducible one-dimensional representations corresponding to four different kinds of fractional branes. The interaction between a stack of $N_{I}(I=1, \ldots, 4)$ branes of type $I$ and a D3-fractional brane, for example of type $I=1$, with an $S U(N)$ gauge field turned on its world-volume, is the sum of four terms

$$
Z=Z_{e}+\sum_{i=1}^{3} Z_{h_{i}}
$$

where each contribution is obtained from the corresponding one in the orbifold $C^{2} / Z_{2}$ by adding an extra factor $1 / 2$, due to the orbifold projection, and an index $i$ that labels the elements of the orbifold group. 
By extracting the coefficient of the term $F^{2}$ and performing on it the field theory limit, one gets the correct running coupling constant and chiral anomalies for $\mathcal{N}=1 \mathrm{SYM}$, which are:

$$
\begin{aligned}
& \frac{1}{g_{Y M}^{2}(\Lambda)}=\frac{1}{g_{Y M}^{2}(\mu)}+\frac{3 N_{1}-N_{2}-N_{3}-N_{4}}{16 \pi^{2}} \log \frac{\Lambda^{2}}{\mu^{2}} \\
& \theta_{Y M}=\left(3 N_{1}-N_{2}-N_{3}-N_{4}\right) \theta .
\end{aligned}
$$

showing again that the supergravity solution, dual to $\mathcal{N}=1 \mathrm{SYM}$ theory, gives the correct answer for the perturbative behavior of the non conformal world volume theory.

\section{References}

[1] P. Di Vecchia, A. Liccardo, R. Marotta and F. Pezzella, Gauge/Gravity Correspondence from Open/Closed String Duality, JHEP 0306 (2003) 007, hep-th/0305061.

[2] P. Di Vecchia and A. Liccardo, Gauge Theories from D Branes, hep-th/0307104 and references therein.

[3] M. Bertolini, P. Di Vecchia and R. Marotta, N=2 four-dimensional gauge theories from fractional branes, hep-th/0112195

[4] M. Bertolini, Four Lectures on the Gauge/Gravity Correspondence, hep-th/0303160.

[5] R. Marotta and F. Sannino, N=1 Super Yang-Mills renormalization schemes for fractional branes, Phys. Lett. B545 (2002) 162, hep-th/0207163.

[6] E. Imeroni and A. Lerda, Non perturbative gauge superpotential from supergravity, hep-th/0310157.

[7] R. G. Leigh and M. Rozali, Brane Boxes, Anomalies, Bending and Tadpoles, Phys. Rev. D59 (1999) 026004, hep-th/9807082.

[8] Z. Kakushadze and R. Roiban, Brane-Bulk Duality and Non-conformal Gauge Theories , JHEP 0103(2001) 043, hep-th/0102125.

[9] R. Marotta, F. Nicodemi, R. Pettorino, F. Pezzella and F. Sannino, N=1 Matter from Fractional Brane, JHEP 0209 (2002) 010, hep-th/0208153.

[10] W. Fischler and L. Susskind, Dilaton Tadpoles, String Condensates and Scale Invariance 2, Phys. Lett. B173(1986) 262 and Dilaton Tadpoles, String Condensates and Scale Invariance, Phys. Lett. B171 (1986)383.

[11] C.G. Callan, C. Lovelace, C. Nappi and S.A. Yost, String loop corrections to beta functions Nucl. Phys. B288 (1987) 525 and Nucl. Phys. B308 (1988) 221.

[12] M. Bertolini, P. Di Vecchia, M. Frau, A. Lerda, R. Marotta and I. Pesando, Fractional D-branes and their gauge duals, JHEP 02 (2001) 014, hep-th/0011077. 
[13] J. Polchinski, $N=2$ gauge-gravity duals, Int. J. Mod. Phys. A16 (2001) 707, hep-th/0011193.

[14] M. Billò, L. Gallot and A. Liccardo, Classical geometry and gauge duals for fractional branes on ALE spaces, Nucl. Phys. B614 (2001) 254, hep-th/0105258

[15] M. Graña and J. Polchinski, Gauge/gravity duals with holomorphic dilaton, Phys. Rev D65 (2002) 126005, hep-th/0106014.

[16] M. Bertolini, P. Di Vecchia, M. Frau, A. Lerda and R. Marotta, N=2 gauge theories on systems of fractional D3/D7 branes, Nucl. Phys. B621 (2002) 157, hep-th/0107057.

[17] M. Bertolini, P. Di Vecchia, G. Ferretti and R. Marotta, Fractional Branes and $N=1$ Gauge Theories, Nucl. Phys. 360 (2002) 222, hep-th/0112187.

[18] M. Bertolini, P. Di Vecchia, M. Frau, A. Lerda and R. Marotta, More anomalies from fractional branes, Phys. Lett. B540 (2002) 104, hep-th/0202195.

[19] M. Bianchi and J.F. Morales, Anomalies \& Tadpoles, JHEP 0003 (2000) 030, hep-th/0002149

[20] M. Bianchi and J.F. Morales, RG-flows and Open/Closed String Duality, JHEP 0008 (2000) 035, hep-th/0006176. 